\title{
Fluorescent Labeling of Silica Gel Powder using Zingiber montanum Extract for a Bright Latent Fingerprint Detection Under UV-Light
}

\author{
KONGSAK PATTARITH ${ }^{1}$, SAKSIT CHANTHAI ${ }^{2}$ and \\ RACHADAPORN BENCHAWATTANANON ${ }^{3 *}$
}

\begin{abstract}
'Department of Chemistry, Faculty of Science, Buriram Rajabhat University, Buriram 31000, Thailand.
${ }^{2,3}$ Materials Chemistry Research Center, Chemistry Division and Center of Excellence for Innovation in CIntegrated Science, Faculty of Science, Khon Kaen University, Khon Kaen 40002, Thailand.

${ }^{*}$ Corresponding author E-mail: rachadaporn@kku.ac.th
\end{abstract}

http://dx.doi.org/10.13005/ojc/370304

(Received: March 20, 2021; Accepted: May 20, 2021)

\begin{abstract}
This research was to study the preparation of fluorescent dust powder to enhance the detection of ultra-bright latent fingerprints using natural plant extract associated with adsorption on silica gel particles by a simple and cheap method environmentally friendly. To study the physical and chemical properties of the fluorescent powder, various factors affecting the latent fingerprints detection including different surface characteristics, temperature, time and overlapping passive fingerprints were investigated. From the results, it was found that the fingerprints on the surface of different materials could be performed and gave good inspection trace, which can be seen with naked eye and appear brightly under UV-light at a wavelength of $366 \mathrm{~nm}$. For the persistence study of the latent fingerprints, it was also found that when kept it in a dark place at room temperature for 30 days, the fingerprints were still visible to the naked eye and glow clearly under UV-light. In addition, the overlapping fingerprints can be clearly identified. Therefore, the study of the fluorescent silica gel dust powder demonstrates good performance and it is a potential use to detect latent fingerprints as a witness in forensic processes.
\end{abstract}

Keywords: Fluorescence, latent fingerprints, Zingiber Montanum extract, Silica gel powder.

\section{INTRODUCTION}

Today's the crimes are becoming more violent and complex. Making the investigation more difficult to arrest the perpetrator latent fingerprints are considered one of the most important evidence in identifying the person present at the scene and can connect to the offender ${ }^{1,2}$. In general, surface fingerprints are divided into three categories: plastic prints, visible prints, and latent prints. The method for examining fingerprints at the scene of the crime will look different. Depending on the fingerprint's compression, Due to different types of pressure marks of the fingerprints on the scene,

This is an Open Access article licensed under a Creative Commons license: Attribution 4.0 International (CC- BY). Published by Oriental Scientific Publishing Company @ 2018

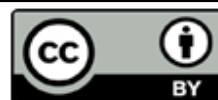


the fingerprints can be noticeable on the nature of the indentation and the length of contact with the surface of various objects, so fingerprints must be selected to suit each type of witnesses. To get a clear and easy fingerprint identification ${ }^{3}$. There are many methods to collect latent fingerprints. Depending on the surface characteristics of the witness material, dusting is a very popular method for detecting latent fingerprints at crime scenes ${ }^{4}$. Because it is a highly effective method can be done easily provides clear fingerprint images, recognized in the justice process $^{5,6}$. Dust is the one of the key factors in this method of detecting latent fingerprint used to sweep away latent fingerprints ${ }^{7}$. There are many types of dust that are used in forensic science. Each has a different property such as color, adhesion and grain size $^{8}$. The ability to stick on the surface of each object is different. It also depends on the weather, humidity, dryness or wetness of the object. Therefore, research studies have been conducted on the preparation of dust used in the detection of latent fingerprints in order to increase the efficiency and reduce the limitations of use ${ }^{9}$. The fluorescent dust is one of the materials being studied for the detection of latent fingerprints ${ }^{10}$. Due to the fluorescence of the material, the fingerprint is cleared and improving the proving procedure ${ }^{11}$ However, various research studies involving the synthesis of fluorescent dust remain limited in many areas, such as the complexity of synthesis ${ }^{11}$. Toxicity of raw materials and dust powder with high price ${ }^{12,13}$ for that reason, it hinders its practical use. Therefore, the research team is interested in a highly effective method of preparing fluorescent dust powder used in the examination of latent fingerprints using dyes extracted from plants that have the ability to glow under UV-light it is applied on the silica gel absorbent material ${ }^{14,15}$. The nature dust powder can be prepared in a simple, inexpensive and environmentally friendly manner ${ }^{16,17}$. The factors affecting latent fingerprints were also studied, such as different material surfaces, temperature, storage times and identification of latent fingerprint from an overlapping fingerprint. In the future, it can be used commercially.

\section{MATERIALS AND METHODS}

\section{Chemicals and characterization}

Silica gel $\left(\mathrm{SiO}_{2}\right)$ and hexane $\left(\mathrm{C}_{6} \mathrm{H}_{14}\right)$ were purchased from Sigma-Aldrich Chemical Co., Ltd.
All the chemicals were used without any further purification. Morphological analysis of the prepared photoluminescence powder was carried out by using Scanning Electron Microscope (LEO/1450). The phase purity and crystal structure were examined by using an X- ray diffractometer (Bruker/D8 Advance). Ultraviolet-visible (UV-Vis) absorption spectra measurements were carried out on UV-Vis diffuse reflectance spectrometer (SHIMADZU/UV-1900). The latent fingerprint images were photographed by using a Smartphone camera.

\section{Preparation of fluorescence labeling powder}

Preparation of fluorescent silica gel powder coated with a phosphor extracted from the Zingiber Montanum (Zingiber) was carried out. The procedure steps were as follows:

\section{Zingiber extract preparation}

The old rhizomes of Zingiberwere cleaned by water rinsing, peeled and then sliced to make the smaller ones. The plant sample (200 g) was extracted with coconut oil $100 \mathrm{~mL}$ at temperature range of $150-200^{\circ} \mathrm{C}$ until the color of Zingiber changing to dark brown and the remaining in the flask was then cooled down to room temperature. After filtered the dark yellow extracted Zingiber oil was obtained.

\section{Preparation of silica gel powder}

Preparation of silica gel powder was done by simple mortar grinding to get its fine powder. It was then sieved through a 325 mesh (44 microns) sieving apparatus. The silica gel powder obtained was packed into a bottle and kept in a desiccator before use.

\section{Fluorescent powder preparation}

Fluorescent powder preparation to be used in the detection of latent fingerprints can be done by weighing $15 \mathrm{~g}$ of the silica gel powder was mixed with $45 \mathrm{~mL}$ Zingiber oil extract, the mixture was magnetically stirred at room temperature for another 12 hours. The mixture was filtered under reduced pressure and the residual solid was washed with hexane to remove coconut oil. The filtrate was evaporated under vacuum apparatus. The prepared fluorescent dust is placed in opaque bottles and also stored in a desiccator. 


\section{Characterization of the synthetic fluorescence powder}

The fluorescent dust powder was mixed with the plant oil extract as mentioned above. Then the mixture was shaken to observe their surface characteristics at the micro level. The particle characteristics were performed using scanning electron microscope (SEM) technique. UV-Visible diffuse reflectance spectroscopy (DRS) technique was used for the latent fingerprint detection test. To measure the light absorption of the resonant fluorescent dust, X-ray diffraction (XRD) technique was used and the fluorescence was tested under UV-light at $366 \mathrm{~nm}$.

In this research, the preparation of fluorescent dust powder was studied for detecting latent fingerprints by dusting the dust onto the fingerprint imprinted in various conditions such as detection of the latent fingerprints on the surface of different materials, the identification of the latent fingerprint from an overlapping fingerprint, the temperature effects and the storage time of the identified fingerprints which can be examined by comparative imaging under both visible light and UV-light at $366 \mathrm{~nm}$.

\section{RESULTS AND DISCUSSION}

\section{Silica gel/Zingiber Montanum extract as fluorescence powder}

The fluorescent labeled silica gel powder which coated with the Zingiber oil extract is shown in Fig. 1. It was tested for fluorescence under UV-light at $366 \mathrm{~nm}$. The results indicate that the obtained fluorescent dust is seen in yellow color, and when it is illuminated under UV-light, it glows clearly. This is caused by the extracts obtained from Zingiber montanum that are adsorbed on the surface of the silica gel powder. Since the oil extract is less polar, this will be beneficial in keeping the dust sticking to the fat in fingerprints. While silica gel itself is polarized, it can bind to the moisture residue in the latent fingerprints.

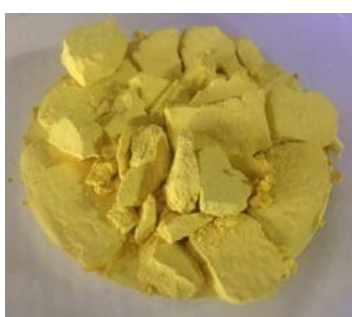

(A)

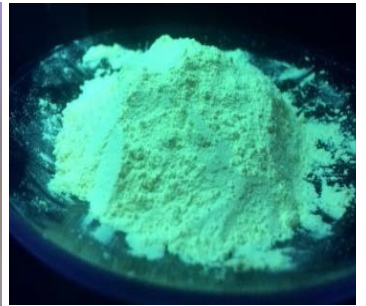

(B)
Fig.1. Fluorescence effect of the fluorescent dust powder, (A) under visible light and (B) Under UV-light

\section{Characterization by UV-Visible diffuse reflectance spectroscopy}

The UV-Visible absorption spectra of pure silica gel powder (line A) compared with its fluorescent dust (line B) are shown in Fig. 2. It was found that the absorbance of pure silica gel powder was low in UVVisible absorption, while the fluorescent dust gave its absorption spectrum in a wavelength of 200-500 $\mathrm{nm}$ with maximum peak approximately at 280 and $420 \mathrm{~nm}$. The absorbed energy excites the electrons from the ground state to the excited state. When the electrons return to their ground state, they release energy in the form of light that visualized as a bright fluorescence under UV-light.

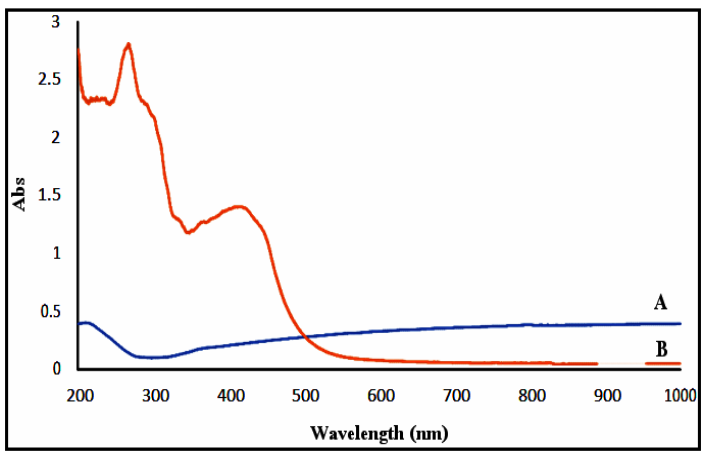

Fig. 2. UV-Vis absorption spectra of pure silica gel powder (A) and the fluorescent dust (B)

Characterization by scanning electron microscope

The surface characteristics of the fluorescent dust compared with its silica gel powder at the micro scale were studied. The fine particles of the fluorescent dust powder are shown in Fig. 3. The morphology of pure silica gel particles (Fig. 3 A) was clearly porous, widely distributed on the surface. When it was adsorbed with the oil extract as the fluorescent dust (Fig. 3 B), the oil extract was adsorbed on the surface making its surface smoother.

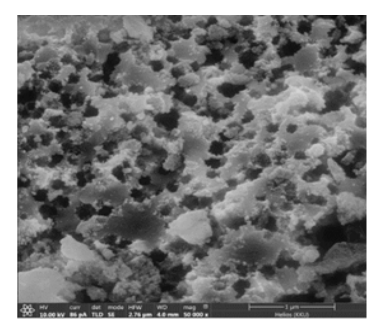

(A)

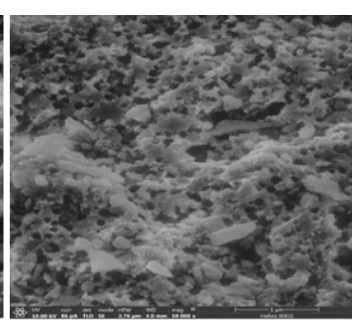

(B)
Fig. 3. SEM images at $50,000 x$ magnification of pure silica gel powder (A) and the fluorescent dust (B) 


\section{Characterization by X-ray diffraction (XRD)} analysis

The metaphorical crystal characterization of the synthetic fluorescent dust comparing with silica gel powder by $\mathrm{X}$-ray diffractometer (XRD) was shown in Fig. 4. The XRD pattern of the fluorescent dust powder is given in Fig. $4 \mathrm{~B}$ and the other one of pure silica gel powder used as a substrate is in Fig. $4 \mathrm{~A}$ representing background with no sign of any crystal particles, indicating the morphology characteristic of an inconsistent solid base.

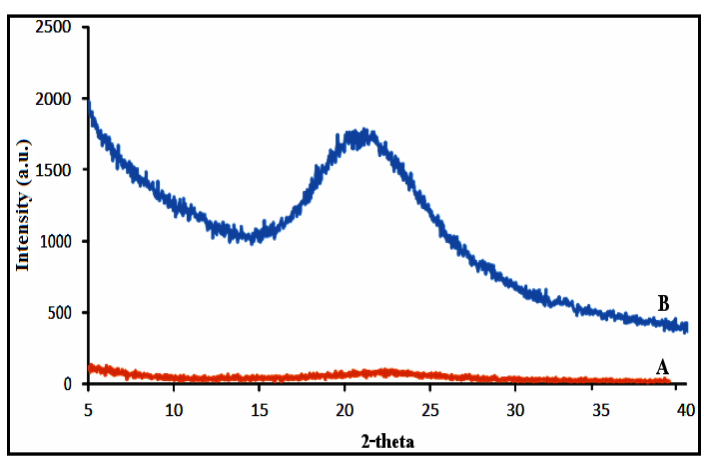

Fig. 4. XRD pattern of pure silica gel powder (A) and the fluorescent dust (B)

Effect of substrate on verification of latent fingerprints with the fluorescent dust

From the examination of hidden fingerprints that are stamped on the surface of various materials with different texture characteristics and then take pictures under simple light and UV-light to compare the contrast of latent fingerprints on the surface of those different materials, the results are shown in Table 1. It was found that the fluorescent dust can adhere to the hidden fingerprint well which can be verified by emitting fluoresce dust under UV at 366 $\mathrm{nm}$. To see the latent fingerprints more quickly, the clarity of the fingerprint depends on the texture, such as the simplicity and the scratch on the surface also depends on the neck and the volume. The store of the owner of the fingerprint is affected as well, which is an individual characteristic concerned.

\section{Effect of retention time of latent fingerprints identified with the fluorescent dust}

To test the durability of the phosphor coating on the silica gel surface, experiments were performed by storing hidden fingerprints on glass panels were examined with fluorescent dust in the dark at room temperature and compared them with the default fingerprint as shown in Table 2. It was found that the storage time affects the fluorescence capacity of the fluorescent dust. It was also found that as the storage time elapsed, the ability of fluorescence under UV-light at $366 \mathrm{~nm}$ decreased. However, when the fingerprint is stored longer for 30 days, even though the fluorescence is reduced but still be able to see the details of the hidden fingerprint clearly.

Table 1: Examination of hidden fingerprints with fluorescent dust on various substrates

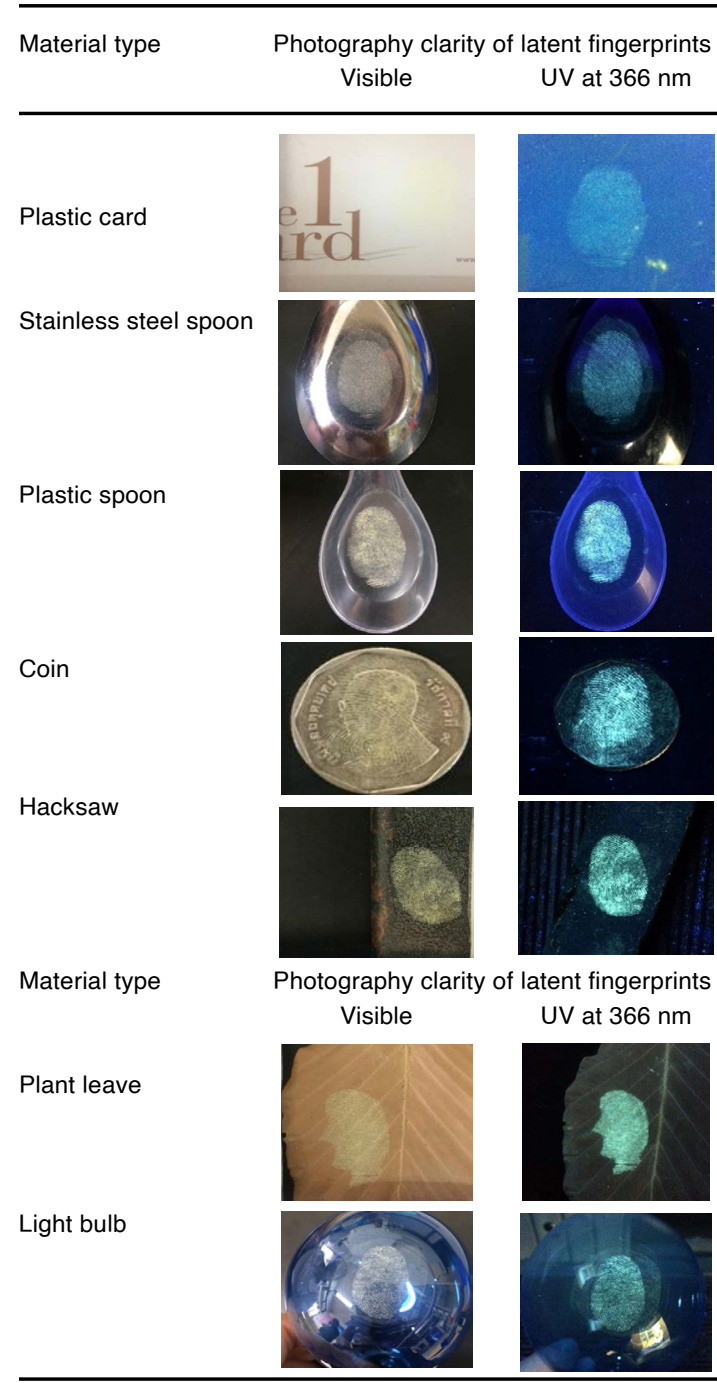

Table 2: Effect of retention time on the contrast of the latent fingerprints

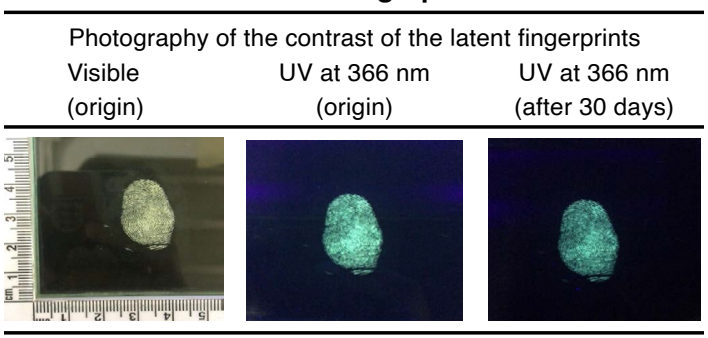




\section{Effect of temperature on storage of latent fingerprints examined with fluorescent dust}

The effect of temperature on the storage of the latent fingerprints was investigated with this fluorescent material. The storage was taken in various temperature for 15 days as shown in Table 3. The study was conducted at temperature of $30^{\circ} \mathrm{C}$, $60^{\circ} \mathrm{C}$ and $90^{\circ} \mathrm{C}$. It was found that the temperature affected on the fluorescence of the dust powder. Because the phosphors are used as the plant extracts. When the fingerprint was stored at higher temperatures, it decreases the glow of the latent fingerprint which is caused by the decay of phosphor if the temperature increases and stores for a long time. The glow will gradually decrease, therefor the storage of verified passive fingerprints must not be stored at high temperature for a long time.

Table 3: Effect of temperature on the storage of latent fingerprints

\begin{tabular}{llll}
\hline Time & \multicolumn{3}{c}{ Temperature (under UV at $366 \mathrm{~nm}$ ) } \\
& $30^{\circ} \mathrm{C}$ & $60^{\circ} \mathrm{C}$ & $90^{\circ} \mathrm{C}$ \\
\hline & & & \\
\hline
\end{tabular}

Verification of latent fingerprints with the fluorescent dust overlay

The effect on latent fingerprint overlays is an important factor for detecting the latent fingerprints. Cause of the target passive fingerprint cannot be determined when there are other passive fingerprints on the surface. Hence, the overlapping passive fingerprint test is one of the key factors in the inspection with fluorescent dust. The results obtained from the study of the superposition of latent fingerprints are shown in Table 4. By examining the hidden fingerprints that are overlapping with the fluorescent dust was observed under UV-light at $366 \mathrm{~nm}$. It was found that the streaks of the latent fingerprints can be clearly seen on both fingerprints, so the fluorescent dust powder can be used as an alternative way to detect the overlapping latent fingerprints.
Table 4: The examination of latent fingerprints with fluorescent dust superposition

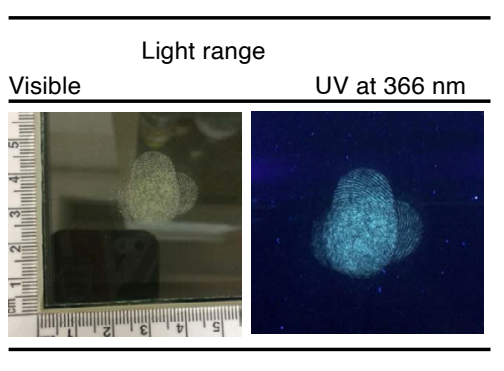

CONCLUSION

From the study, the fluorescent dust powder was prepared from silica gel powder coated with an oil extract of Zingiber and used to detect the latent fingerprints which normally applied in forensic science. It was found that the fluorescence property was bright signal under UV-light at $366 \mathrm{~nm}$. Their factors affecting the detection and storage of latent fingerprints were investigated in details such as different types of surface substrate materials, fingerprint overlaying, time duration and temperature of the fingerprint storage. It was also found that different types of the substrate materials used have different textures to be able to verify the hidden fingerprints with this fluorescent dust and more clearly seen when it was observed under UV-light. The storage temperature can affect the glowing of the fluorescent dust, since the natural phosphor in the plant extract will getting decompose if it is exposed to high temperatures over a long time. This will also reduce the fluorescence intensity of the passive fingerprint that has been verified.

\section{ACKNOWLEDGEMENT}

The authors thank financial support from Integrated Science, Faculty of Science, Materials Chemistry Research Center (MCRC) and Department of Chemistry \& Center of Excellence for Innovation in Chemistry (PERCH-CIC), Faculty of Science, Khon Kaen University, Thailand.

\section{Conflict of interest}

The authors have declared no conflict of interest. 


\section{REFERENCES}

1. Parka, J.P.; Parka, S.J.; Kwakb, M., \& Yanga, H.K., Rapid visualization of latent fingerprints with Eu-doped $\mathrm{La}_{2} \mathrm{Ti}_{2} \mathrm{O}_{7}$. J. Lumin., 2018, 201, 275-283.

2. Wang, W.; Lei, X.; Ye, Z.; Zhao, N.; \& Yang, $\mathrm{H}$., The luminescent properties and latent fingerprint identification application of AIN: Ce, Tb phosphors. J. Alloys Compd., 2017, 705, 253-261.

3. Park, J.P.; Chung, J.W., \& Yang, H.K., Versatile fluorescent $\mathrm{Gd}_{2} \mathrm{MoO}_{6}: \mathrm{Eu}^{3+}$ nanophosphor for latent fingerprints and anti-counterfeiting applications. Ceram. Int., 2019, 45, 11591 11599.

4. Sodhi, G.S.; \& Kaur, J.; Power method for detecting latent fingerprint: a review Forensic Sci. Int., 2001, 120, 172-176.

5. Yuana, C.; Lia, M.; Wanga, M.; Zhanga, X.; Yina, Z.; Songa, K., \& Zhanga, Z., Sensitive development of latent fingerprints using Rhodamine B-diatomaceous earth composites and principle of efficient image enhancement behind their fluorescence characteristics. Chem. Eng. J., Article in press.

6. Navamia, D.; Basavaraja, R.B.; Darshanb, G.P.; Hajeebaba, K.; Inamdara, S.C.; Sharmac, H.B., \& Nagabhushana, H., Evolution of shapes and identification of level II and III features of fingerprints using $\mathrm{CaZrO}_{3}: \mathrm{Sm}^{3+}$ fluorescent markers prepared via solution combustion route. Opt. Mater., 2019, 88, 479-487.

7. Prabakaran, E.; \& Pillay, K.; Synthesis and characterization of fluorescent $\mathrm{N}$-CDs/ ZnONPs nanocomposite for latent fingerprint detection by using powder brushing method. Arab. J. Chem., 2020, 13, 3817-3835.

8. Li, H.; Guo, X.; Liu. J.; \& Li. F.; A synthesis of fluorescent starch based on carbon nanoparticles for fingerprints detection. Opt. Mater., 2016, 60, 404-410.

9. Park, S.J.; Park, J.Y.; \& Yang, H.K.; Luminescence of a novel cyan emitting $\mathrm{Sr}_{10}\left(\mathrm{PO}_{4}\right)_{6} \mathrm{O}: \mathrm{Ce}^{3+}$ phosphor for visualization of latent fingerprints and anti-counterfeiting Applications. Sens. Actuators B Chem., 2018,
262, 542-554.

10. Fouad, R., \& Saif, M., Synthesis, spectroscopic and photoluminescence studies of novel Eupnanophosphor complex as fluorescent sensor for highly sensitive detection of latent fingerprints and anti-counterfeiting. J. Mol. Struct., 2020, 1217, 128472.

11. Prabakaran, E., \& Pillay, K., Synthesis and characterization of fluorescent Europium (III) complex based on D-dextrose composite for latent fingerprint detection. J. Saudi Chem. Soc., 2020, 24, 584-605.

12. Basavaraj, R.B.; Nagabhushana, H.; Darshan, G.P.; Prasad, B.D.; Rahul, M.; Sharma, S.C.; Sudaramani, R., \& Archana, K.V., Red and green emitting CTAB assisted $\mathrm{CdSiO}_{3}: \mathrm{Tb}_{3} \mathrm{p} /$ $\mathrm{Eu}_{3} \mathrm{p}$ nanopowders as fluorescent labeling agents used in forensic and display applications. Dyes Pigm., 2017, 147, 364-377.

13. Li, B.-Y.; Zhang, X.-L.; Zhang, L.-Y.; Wang T.-T.; Li, L.; Wang, C.-G., \& Su, Z.M.,WIRresponsive $\mathrm{NaYF}_{4}: \mathrm{Yb}, \mathrm{Er}, \mathrm{Gd}$ fluorescent upconversion nanorods for the highly sensitive detection of blood fingerprints. Dyes Pigm., 2016, 134, 178-185.

14. Saif, M.; Alsayed, N.; Mbarek, A.; Kemary, M.-E., \& Mottaleb, M.S.A. Preparation and characterization of new photoluminescent nano-powder based on $\mathrm{Eu}_{3} \mathrm{p}: \mathrm{La}_{2} \mathrm{Ti}_{2} \mathrm{O}_{7}$ and dispersed into silica matrix for latent fingerprint detection. J. Mol. Struct., 2016, 1125, 763-771.

15. Li, F.; Li, H., \& Cui, T., (2017). One-step synthesis of solid state luminescent carbonbased silica nanohybrids for imaging of latent fingerprints. Opt. Mater., 2017, 73, 459-465.

16. Bhagat, D.S.; Suryawanshi, I.V.; Gurnule, W.B.; Sawant, S.S., \& Chavan, P.B., Greener synthesis of $\mathrm{CuO}$ nanoparticles for enhanced development of latent fingerprints. Mater. Today Commun., 2020, Article in press.

17. Xu, S.; Shen, J.; Wang, Z.; Du, J.; Wang, C.; Zhao, Z.; \& Cui, Y.; One-step Synthesis of Stable, Nontoxic, Orange Quantum Dots Fluorescent. Nanomater. Nanotechnol., 2016, 6, 25. 